\title{
Binocular Perception of 2D Lateral Motion and Guidance of Coordinated Motor Behavior
}

\author{
Aaron J. Fath ${ }^{1 *}$, Winona Snapp-Childs ${ }^{1}$, Georgios K. Kountouriotis ${ }^{2}$, \\ and Geoffrey P. Bingham ${ }^{1}$ \\ ${ }^{1}$ Department of Psychological and Brain Sciences, Indiana University \\ ${ }^{2}$ Department of Psychology, Manchester Metropolitan University \\ ${ }^{*}$ Corresponding author: ajfath@indiana.edu \\ This article may not exactly replicate the final version published in Perception. It is not the \\ copy of record. \\ (C2015. This manuscript version is made available under the CC-BY-NC-ND 4.0 license \\ http://creativecommons.org/licenses/by-nc-nd/4.0/
}

\begin{abstract}
Zannoli, Cass, Alais, and Mamassian (2012) found greater audiovisual lag between a tone and disparity-defined stimuli moving laterally $(90-170 \mathrm{~ms})$ than for disparity-defined stimuli moving in depth or luminance-defined stimuli moving laterally or in depth (50-60 ms). We tested if this increased lag presents an impediment to visually-guided coordination with laterally moving objects. Participants used a joystick to move a virtual object in several constant relative phases with a laterally oscillating stimulus. Both the participant-controlled object and the target object were presented using a disparity-defined display that yielded information through changes in disparity over time (CDOT) or using a luminance-defined display that additionally provided information through monocular motion and interocular velocity differences (IOVD). Performance was comparable for both disparity-defined and luminance-defined displays in all relative phases. This suggests that, despite lag, perception of lateral motion through CDOT is generally sufficient to guide coordinated motor behavior.

Keywords: coordination, stereomotion, binocular vision, motion perception
\end{abstract}

\section{INTRODUCTION}

The study of perception not only seeks to answer how organisms are able to correctly interpret information, but also how this information is used in such a way that it can guide coherent, stable, and effective behavior. Because of the dynamic nature of the environment, understanding the visual perception of motion is critical to this end. Motion perception displays significant redundancy of function, as do many other forms of perception. As conditions change during any perceptual function, a source of information may become absent or degraded, so a system that relies on multiple sources is more flexible and stable. This redundancy 
can be seen throughout human vision and visually-guided motor control, such as in targeted walking (Warren, Kay, Zosh, Duchon, \& Sahuc, 2001), affordance perception (Wraga \& Proffitt, 2000), and size perception (Dixon, Wraga, Proffitt, \& Williams, 2000). In the area of motion perception, the speed and direction of optic flow caused by object translation is a monocularly-available cue for object motion. Although binocular disparity is primarily thought of as a depth cue, it also plays a role in motion perception. Decades ago, these two cues were identified as the bases for two potential high-order sources of information for perception of motion: (a) differences between the optic flow of the two eyes, known as interocular velocity differences (IOVD), and (b) changes in binocular disparity over time (CDOT) (Beverley \& Regan, 1973; Rashbass \& Westheimer, 1961; Regan \& Beverley, 1973).

More recent work has confirmed the use of IOVD and CDOT for perception of motion and has tried to define their roles more clearly (Harris, Nefs, \& Grafton, 2008; Rokers, Cormack, \& Huk, 2009). Zannoli et al. (2012) performed a study that measured the processing latencies for IOVD and CDOT during different kinds of motion. This was done by comparing the time required to process motion when viewing disparity-defined stimuli to the time required for stimuli defined by both luminance and disparity. They measured the time lag at which participants perceived the oscillations of the amplitude of a tone to be inphase with the oscillations of movement of visual stimuli that were either moving laterally or solely in depth. They found that perception of disparity-defined visual stimuli moving laterally lagged behind perception of the auditory tone by 90-170 ms, but only by $\sim 60 \mathrm{~ms}$ for disparity-defined stimuli moving in depth. The lag for luminance-defined stimuli was $\sim 51 \mathrm{~ms}$ when moving laterally and $\sim 43 \mathrm{~ms}$ when moving in depth.

The finding that lateral motion of disparity-defined stimuli was processed slower raises the question of how suited CDOT information is for tracking laterallymoving objects. The visual system might rely entirely on monocular flow and IOVD information for laterally moving objects when it is available because the lag from CDOT is prohibitive in those conditions. Of course, object motion is rarely purely lateral, but it could be the case that the lateral component of $3 \mathrm{D}$ object motion is perceived without using CDOT. However, in principle, a CDOTonly motion perception system could still function near-optimally in guidance of most day-to-day behavior, even in the rare instances that involve purely lateral movement. If all motion is perceived with the same lag, coordinated behaviors such as typing, reaching, or sewing should not be affected. Even interactions with independently-moving objects, such as when grasping a rolling ball, might not be problematic unless an object is moving unpredictably. However, whether or not performance would degrade for interactions with laterally-moving stimuli in the absence of monocular motion and IOVD is an empirical matter.

The purpose of this study was to determine if CDOT information is sufficient to guide coordination with purely lateral motion. To test if the increased lag found by Zannoli et al. (2012) presents enough of an impediment to affect visually-guided coordination with laterally-oscillating stimuli, we tested how people performed in a rhythmic coordination task with an independently-moving object in lateral motion, both when the stimulus was only defined by disparity and when it was defined by both disparity and luminance. If we found decreased performance with the disparity-defined stimuli, we could conclude that CDOT is not sufficient, likely due to increased visual lag. There is a task that has been studied substantially in 

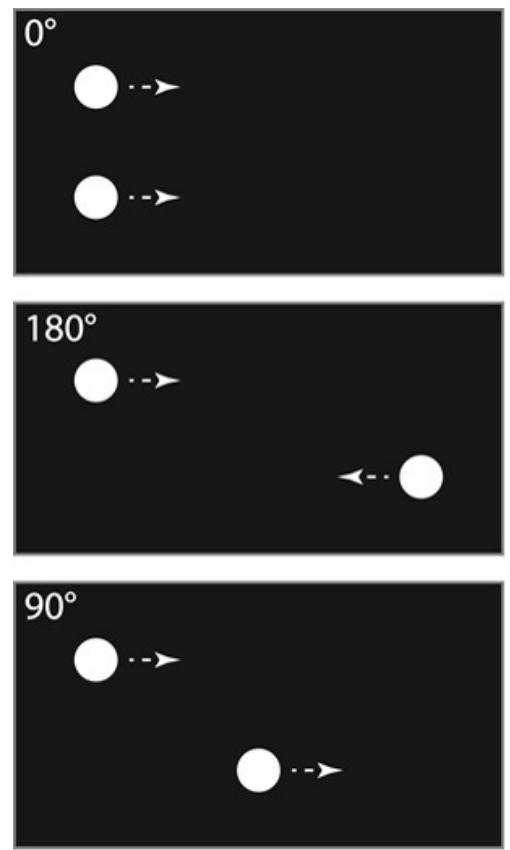

Figure 1 Illustration of the three relative phase relations used. Each shows the top, computer-controlled circle at the leftmost possible location beginning rightward movement, and the bottom circle at the corresponding location it would need to be to produce the target relative phase.

the coordination literature that consists of oscillatory lateral motion. Participants view a filled circle oscillating laterally on a screen and use a joystick to move another circle in a specified target relative phase with the computer-controlled circle (Wilson, Collins, \& Bingham, 2005). We have adapted this task for use in the current study, using target relative phases of $0^{\circ}$ (inphase), $180^{\circ}$ (antiphase), and $90^{\circ}$ (Figure 1). The task was performed with both luminance-defined and disparity-defined stimuli. Luminance-defined stimuli provided participants with monocular optic flow, the resulting IOVD information, and CDOT, but disparitydefined stimuli only provided participants with CDOT information. If CDOT information is sufficient to guide coordination in this task, we should see no difference in performance between these two conditions. Performing $90^{\circ}$ is more difficult than $180^{\circ}$, which is in turn more difficult than $0^{\circ}$. It could be the case that increased lag of disparity-defined stimuli is not problematic for easier relative phases, but is for more difficult ones. Using multiple relative phases allowed us to test if task difficulty affected participants more when coordinating with disparity-defined stimuli.

\section{RESULTS}

All participants were previously trained in the task during a prior study using luminance-defined stimuli, so we expected competent performance in that visual condition. We expected participants to correctly perceive the disparity-defined stimuli and for their resulting performance to be comparable to their performance 


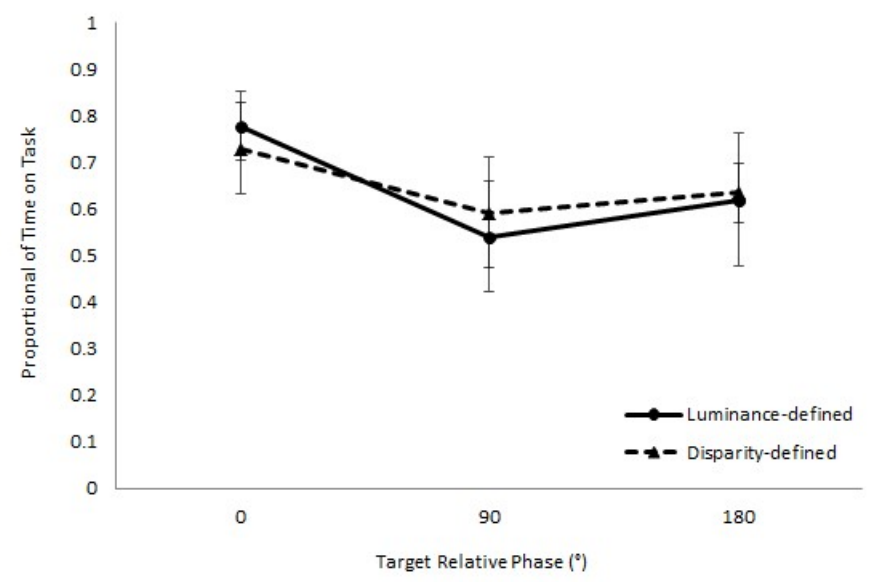

Figure 2 Performance across target relative phase for both stimulus types. Being on task

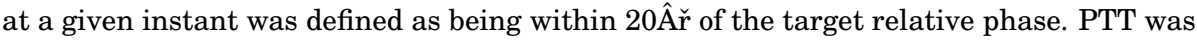
averaged across participants in each condition (luminance-defined and disparity-defined) for each target relative phase $\left(0^{\circ}, 90^{\circ}\right.$, and $\left.180^{\circ}\right)$. The stimuli in the luminance-defined condition provided both interocular velocity differences (IOVD) and change in disparity over time (CDOT). The stimuli in the disparity-defined condition provided only CDOT information. Error bars represent standard deviation.

with the luminance-defined stimuli. Participants performing a relative phase coordination task typically perform best when attempting to produce $0^{\circ}$, followed by $180^{\circ}$, and then the intermediate phase of $90^{\circ}$ (Kelso, 1984). Thus, we expected performance as measured by proportion of time on task (PTT) to be best when attempting to produce $0^{\circ}$ relative phase. With feedback, participants can train to perform $90^{\circ}$ as well as they can perform $180^{\circ}$, but still not as well as $0^{\circ}$ (Wilson, Snapp-Childs, Coats, \& Bingham, 2010). Thus, we expected performance of $180^{\circ}$ and $90^{\circ}$ to not be as high as for $0^{\circ}$, but still good because participants had previous training. This was predicted for both types of stimuli.

Figure 2 shows performance for both stimuli, in all target relative phases, measured as PTT within $20^{\circ}$ of the target relative phase. The figure shows that participants performed comparably in the disparity-defined and luminancedefined conditions. In addition, as predicted, participants performed comparably in the $90^{\circ}$ and $180^{\circ}$ conditions, but not as well as they did in the $0^{\circ}$ condition. We performed a two-way repeated measures ANOVA with stimulus type (luminancedefined and disparity-defined) and target relative phase $\left(0^{\circ}, 90^{\circ}\right.$, and $\left.180^{\circ}\right)$ as factors. There was a main effect of target relative phase $(F(2,22)=20.55, p<.001)$, but no main effect of stimulus type and no interaction.

\section{DISCUSSION}

Perception operates in the service of action. The implications of the study of perception are best understood in the context of how they bear on interaction with a dynamically-changing environment. To execute stable behavior, the human visual system relies on multiple sources of information about motion, although one source is sufficient under most conditions. In the current study we show that, 
under stable conditions, CDOT information is sufficient to guide perception of, and coordination with, laterally moving objects. This is true despite the CDOT motion perception system having greater processing latencies for lateral motion than the IOVD system.

Continued work on the functional roles of IOVD and CDOT are of particular importance due to the apparent differences in how the IOVD and CDOT motion perception systems age, especially in light of the aging population. Significant deficits in monocular motion perception are experienced by older adults (Norman, Ross, Hawkes, \& Long, 2003; Wist, Schrauf, \& Ehrenstein, 2000), and those in this population are often wholly unable to perceive depth and shape when specified by differential motion and static binocular disparity (Norman, Dawson, \& Butler, 2000), especially in the presence of noise (Norman et al., 2012). However, these can be perceived accurately with dynamic binocular disparity, even in the presence of noise (Norman et al., 2006, 2012), except under the most difficult of viewing conditions (e.g. high disparity combined with high spatial frequency) (Norman et al., 2000, 2008). However, in order to understand how the IOVD and CDOT motion perception systems degrade with age, we must understand how these systems work in normally-functioning visual systems.

Evidence suggests that motion perception with disparity-based information is a slower process than motion perception with flow-based information, even for motion-in-depth (Regan \& Beverley, 1973; Tyler, 1971). It is not surprising, then, that the perception of the times-to-contact of fast-moving objects is more accurate for stimuli that only provide monocular motion and IOVD than for stimuli that only provide CDOT (Fath, Lind, \& Bingham, 2015). Interestingly, this study also shows that perception of slow-moving objects is more accurate for stimuli that only provide CDOT than those that provide monocular motion and IOVD. In this way, the spatiotemporal domains of the IOVD and CDOT systems appear to be clearly demarcated. However, the current study shows that this is not the case for their spatial domains, despite small differences in processing latencies under certain highly-constrained conditions.

\section{METHODS}

\section{Participants}

Twelve adults (five female and seven male, aged 25-36 years) were recruited to participate in this study. The participants had normal or corrected-to-normal vision, with stereoacuity of at least 80 arcsec crossed disparity as measured by the Stereo Fly Test (Stereo Optical Company, Inc.). All participants had previously been trained in the task as part of their participation in a prior study. This was done because many people are unable to reliably produce $90^{\circ}$ relative phase without training, regardless of viewing conditions. All participants gave their informed consent prior to participation. All procedures were approved by and conform to the standards of the Indiana University Institutional Review Board.

\section{Procedure}

The procedure was nearly identical to those used by Coats, Snapp-Childs, Wilson, and Bingham (2013) and by Coats, Wilson, Snapp-Childs, Fath, and Bingham (2014). Each stimulus was displayed on a Dell UltraSharp LCD monitor with a 
resolution of $1920 \times 1080$ and a refresh rate of $60 \mathrm{~Hz}$, viewed from a distance of $76.2 \mathrm{~cm}$. There was no source of light in the room besides the monitor. A Logitech Force 3D Pro joystick (force feedback feature disabled) was attached via USB. The joystick was placed off to the side of the display that corresponded to the participant's dominant hand and was controlled by that hand. The computer presented a display showing two filled circles, one above the other, against a black background. During task demonstrations, both circles were under the control of the computer. During trials, the top circle was under the control of the computer and the bottom was under the control of the participant via the joystick. Computer controlled circles in both instances oscillated laterally with a frequency of $0.75 \mathrm{~Hz}$. The amplitude of this movement was $15 \mathrm{~cm}$ and each circle was $3 \mathrm{~cm}$ in diameter. Stimulus presentation, data recording, and all data analysis were handled by a custom Matlab toolbox, incorporating the Psychtoolbox (Brainard, 1997).

Participants first viewed an $8 \mathrm{~s}$ task demonstration of the two circles in $0^{\circ}$ relative phase. Then they attempted to produce and maintain $0^{\circ}$ relative phase for five $20 \mathrm{~s}$ trials, the first of which was a practice trial and was not analyzed. This was done by moving the joystick from side to side to control the bottom circle and coordinate its phase with that of the computer-controlled top circle. This procedure was then repeated for the $180^{\circ}$ and $90^{\circ}$ target relative phase conditions. All participants performed this block of three target relative phases using two different types of stimuli, one that provided participants with monocular motion, IOVD, and CDOT (luminance-defined), and one that only provided participants with CDOT (disparity-defined). Both the luminance-defined block and the disparity-defined block were performed in the same session, with the order of blocks counterbalanced across participants.

In the luminance-defined condition, the circles were white on a black background. The disparity-defined stimuli were red and blue dynamic random-dot stereograms, known as kinematograms, viewed with anaglyph glasses. These displays consisted of a square pattern of red dots and an identical pattern of blue dots with a small lateral offset. This dot cloud was $15 \times 15 \mathrm{~cm}$ and consisted of 25,000 dots. When viewed through the anaglyph glasses, only one of these two patterns was visible to each eye, and because the patterns were identical besides this offset, each matching pair of red and blue points was perceived as a single point. The lateral offset of each pair of matching red and blue points defined the binocular disparity of each resulting perceived single point. Most of the square defined a background at screen depth. For each frame, the correct location of the circles was determined and then the disparity of all points within these circles was manipulated to specify a depth of $5 \mathrm{~cm}$ in front of the screen. This difference in disparity of points is all that defined the circle. Thus, any given frame viewed monocularly looked like an array of random points. The circles could only be perceived when viewed binocularly with the anaglyph glasses on. Because each frame presented a completely new pattern of random dots, no coherent monocular motion existed across frames.

\section{Data Analysis}

The position time series for both circles from each trial were filtered using a low-pass Butterworth filter with a cut-off frequency of $10 \mathrm{~Hz}$. These position time series were numerically differentiated to yield velocity time series for each circle. These were used to compute time series of relative phase, the key measure of 
coordination between the two circles of a given trial. To assess the stability of coordination over the course of a trial, we measured the proportion of time on task (PTT). A participant was determined to be on task at a given instant if the relative phase of the two circles at that moment fell within a $\pm 20^{\circ}$ window of the target relative phase. We averaged PTT, for each participant, over the trials performed in a given condition.

We chose PTT as the primary performance measure because, in human movement, stability is not independent of mean relative phase. Thus, overall movement variability (e.g. the standard deviation of mean relative phase or mean vector length) is confounded with the actual relative phase produced. Coordination stability at $90^{\circ}$ can be artificially elevated if participants spend time producing some other relative phases. This is a common occurrence because the $0^{\circ}$ and $180^{\circ}$ relative phases are natural attractors (Kelso, 1984; Wilson et al., 2005; Zanone \& Kelso, 1992). Using PTT as a performance measure allowed us to address this problem (Coats et al., 2014; Snapp-Childs, Wilson, \& Bingham, 2011). We performed a two-way repeated measures ANOVA with stimulus type (luminancedefined and disparity-defined) and target relative phase $\left(0^{\circ}, 180^{\circ}\right.$, and $\left.90^{\circ}\right)$ as factors.

CONFLICT OF INTEREST The authors declared no potential conflicts of interest with respect to the research, authorship, and/or publication of this article.

FUNDING The authors received no financial support for the research, authorship, and/or publication of this article.

\section{REFERENCES}

Beverley, K. I., \& Regan, D. (1973). Evidence for the existence of neural mechanisms selectively sensitive to the direction of movement in space. The Journal of Physiology, 235(1), 17-29.

Brainard, D. H. (1997). The Psychophysics Toolbox. Spatial vision, 10(4), 433-6.

Coats, R. O., Snapp-Childs, W., Wilson, A. D., \& Bingham, G. P. (2013). Perceptuomotor learning rate declines by half from 20s to 70/80s. Experimental Brain Research, 225(1), 75-84.

Coats, R. O., Wilson, A. D., Snapp-Childs, W., Fath, A. J., \& Bingham, G. P. (2014). The 50s cliff: perceptuo-motor learning rates across the lifespan. PloS one, $9(1)$, e85758.

Dixon, M. W., Wraga, M., Proffitt, D. R., \& Williams, G. C. (2000). Eye height scaling of absolute size in immersive and nonimmersive displays. Journal of Experimental Psychology: Human Perception and Performance, 26(2), 582-93.

Fath, A. J., Lind, M., \& Bingham, G. P. (2015). Stereomotion perception of time-tocontact using multiple sources of binocular motion information. Manuscript submitted for publication.

Harris, J. M., Nefs, H. T., \& Grafton, C. E. (2008). Binocular vision and motion-indepth. Spatial Vision, 21(6), 531-47.

Kelso, J. A. (1984). Phase transitions and critical behavior in human bimanual coordination. Am J Physiol Regulatory Integrative Comp Physiol, 246(6), R1000-1004. 
Norman, J. F., Crabtree, C. E., Herrmann, M., Thompson, S. R., Shular, C. F., \& Clayton, A. M. (2006). Aging and the perception of 3-D shape from dynamic patterns of binocular disparity. Perception \& Psychophysics, 68(1), 94-101.

Norman, J. F., Dawson, T. E., \& Butler, A. K. (2000). The effects of age upon the perception of depth and 3-D shape from differential motion and binocular disparity. Perception, 29(11), 1335-1359.

Norman, J. F., Holmin, J. S., Beers, A. M., Cheeseman, J. R., Ronning, C., Stethen, A. G., \& Frost, A. L. (2012). Aging and the discrimination of 3-D shape from motion and binocular disparity. Attention, Perception \& Psychophysics, 74(7), 1512-21.

Norman, J. F., Norman, H. F., Craft, A. E., Walton, C. L., Bartholomew, A. N., Burton, C. L., ... Crabtree, C. E. (2008). Stereopsis and aging. Vision Research, 48(23-24), 2456-65.

Norman, J. F., Ross, H. E., Hawkes, L. M., \& Long, J. R. (2003). Aging and the perception of speed. Perception, 32(1), 85-96.

Rashbass, C., \& Westheimer, G. (1961). Disjunctive eye movements. The Journal of Physiology, 159, 339-60.

Regan, D., \& Beverley, K. I. (1973). Some dynamic features of depth perception. Vision Research, 13(12), 2369-79.

Rokers, B., Cormack, L. K., \& Huk, A. C. (2009). Disparity- and velocity-based signals for three-dimensional motion perception in human MT+. Nature Neuroscience, 12(8), 1050-5.

Snapp-Childs, W., Wilson, A. D., \& Bingham, G. P. (2011). The stability of rhythmic movement coordination depends on relative speed: the Bingham model supported. Experimental Brain Research, 215(2), 89-100.

Tyler, C. W. (1971). Stereoscopic Depth Movement: Two Eyes Less Sensitive than One. Science, 174(4012), 958-961.

Warren, W. H., Kay, B. A., Zosh, W. D., Duchon, A. P., \& Sahuc, S. (2001). Optic flow is used to control human walking. Nature Neuroscience, 4(2), 213-6.

Wilson, A. D., Collins, D. R., \& Bingham, G. P. (2005). Perceptual coupling in rhythmic movement coordination: stable perception leads to stable action. Experimental Brain Research, 164(4), 517-28.

Wilson, A. D., Snapp-Childs, W., Coats, R., \& Bingham, G. P. (2010). Learning a coordinated rhythmic movement with task-appropriate coordination feedback. Experimental Brain Research, 205(4), 513-20.

Wist, E. R., Schrauf, M., \& Ehrenstein, W. H. (2000). Dynamic vision based on motion-contrast: changes with age in adults. Experimental Brain Research, 134(3), 295-300.

Wraga, M., \& Proffitt, D. R. (2000). Mapping the zone of eye-height utility for seated and standing observers. Perception, 29(11), 1361-1383.

Zannoli, M., Cass, J., Alais, D., \& Mamassian, P. (2012). Disparity-based stereomotion detectors are poorly suited to track 2D motion. Journal of Vision, 12(11), 15.

Zanone, P. G., \& Kelso, J. A. (1992). Evolution of behavioral attractors with learning: nonequilibrium phase transitions. Journal of Experimental Psychology: Human Perception and Performance, 18(2), 403-21. 